\title{
If Certain Foods are Addictive, How Might this Change the Treatment of Compulsive Overeating and Obesity?
}

\author{
Caroline Davis • Jacqueline C. Carter
}

Published online: 6 March 2014

(C) Springer International Publishing AG 2014

\begin{abstract}
This review summarizes the evidence - both current and from an historic perspective - that many processed foods, specifically those in which the palatability has been enhanced with sugar, fat, and salt, have addictive properties similar to drugs such as nicotine, alcohol, and stimulants. The addictive potential of these foods thereby adds to a growing acceptance of 'food addiction' as a viable clinical entity and an important area for further investigation. The evidence that some cases of binge eating disorder can best be conceptualized as a food addiction also has important treatment implications for those suffering from compulsive overeating. This review also discusses the utility of interventions such as motivational interviewing, psychoeducational programs focused on the neurobiologic aspects of excessive consumption of hyper-palatable foods, and the development of cognitive behavioral strategies to increase an individual's ability to tolerate food cravings as temporary states, and better inhibit urges to overeat.
\end{abstract}

Keywords Behavioral addictions - Addictive foods . Compulsive overeating · Obesity · Treatment $\cdot$ Interventions · Food addiction $\cdot$ Binge eating disorder $\cdot$ Motivational interviewing $\cdot$ Psycho-education programs $\cdot$ Cravings · Inhibitory control $\cdot$ Hyper-palatable foods

C. Davis $(\bowtie)$

School of Kinesiology \& Health Science, York University, 343

Bethune College, 4700 Keele Street, Toronto, ON M3J1P3, Canada

e-mail: cdavis@yorku.ca

J. C. Carter

Department of Psychology, Memorial University of Newfoundland,

St. Johns, NL, Canada

\section{Introduction}

Not until the late 19th century did the term addiction enter the lexicon used to describe excessive use of inebriating substances $^{1}$. At that time, it mostly described a dependence on drugs other than alcohol [2]. Interestingly, the first use of the word addiction in the Quarterly Journal of Inebriety [QJI], the original addiction-medicine journal, appeared in 1890 in reference to chocolate [2]. Since then, the notion that certain foods have addictive properties has had a long, albeit somewhat inconsistent and interrupted, history. Several early allusions to the addictive properties of "stimulating" foods were found in other 19th century issues of QJI:

"It is a fact that some foods are more stimulating to the brain cortex than others, e.g. strong beef tea than milk, flesh than bread ... If from childhood ... a brain has depended on stimulating diet and drink for its restoration when exhausted, there is an intense and irresistible craving set up for such food and drink stimulants whenever there is fatigue. Such a brain has developed an affinity for them, and for such alone. Milk and farinaceous diet often become repugnant, and when taken do not satisfy the craving. Its owner becomes physiologically a flesh-eater and an alcohol-drinker" [3 pg. 207].

And,

"If we now look at the effects of the nerve stimulants nearest food, viz., tea and coffee and cocoa: the custom

\footnotetext{
${ }^{1}$ It is particularly noteworthy that more than a century elapsed before the American Psychiatric Association formally adopted the term in 2013. In the Diagnostic and Statistical Manual. Fifth Edition [DSM-5] [1], "Addictions and Related Disorders" replaced the "Substance Abuse" and "Substance Dependence" categories found in previous versions of the DSM.
} 
of taking these for the dissipation of the feeling of exhaustion sets up in brains of the keenly-working but unstable type a commanding craving and a dominant impulse in many cases. The chemical constitution of all such substances has an affinity for the highest centers in the brain, stimulating and soothing some of them in a very subtle way, most delightful to the consciousness of their owners" [3 pg. 208].

The seductive allure of chocolate has a centuries-old legacy beginning with the discovery of cocoa seeds in Mayan Central America, to their cultivation in Africa, followed by its production — after being suitably sweetened with sugar — and eventual introduction into European culture as a novel and fascinating consumer product [4]. Undoubtedly, chocolate has been one of the most celebrated foods since its initial popularity in the 17 th century when it was variously promoted as "medicinal, excitingly foreign, and pleasurable" and "strongly associated with leisure and decadence" [5 pg. 27]. It was not, however, until the latter half of the 20th century that chocolate became an emerging, although relatively short-lived, focus in human addiction-related research. For instance, among selfproclaimed "chocoholics", there was considerable agreement that this confection is habit forming, elicits frequent and pronounced cravings, produces strong and immediate feelings of well-being, and that during periods of abstinence there are pronounced symptoms of withdrawal $[6,7]$. It was also during this time period that self-help organizations began to appear, based on the 12-step program developed for Alcoholics Anonymous, to aid those with compulsive patterns of overeating in response to foods such as chocolate - reinforcing the view, at least among the general public, that hyper-palatable foods have addictive properties, and that for some individuals, overeating can be an addictive behavior. Overeaters Anonymous was established as early as 1960, with Compulsive Eaters Anonymous and Food Addicts Anonymous following a few decades later in 1985 and 1987, respectively.

By the end of the century, it was recognized that chocolate contained special properties, in addition to its hedonic appeal (i.e., the texture, palatability, and aroma), that evoked responses and compulsive behaviors similar to those elicited by other addictive substances. In susceptible individuals, the psychoactive ingredients in chocolate (methylxanthines, biogenic amines, and cannabinoid-like fatty acids) were thought to regulate mood by increasing levels of serotonin and dopamine [8]. Such recognition prompted a handful of studies comparing "Chocolate Addicts" to control participants on addiction-related affective and physiologic responses to chocolate consumption, and chocolate-related cues. For instance, the Chocolate Addicts demonstrated greater arousal, more pronounced cravings, more negative affect, and consumed more chocolate than controls [9]. They also demonstrated more aberrant eating behaviors and attitudes in general. Such findings led to the conclusion that excessive motivation for, and consumption of, chocolate could be considered a valid clinical parallel with addiction in general. As is the case today, however, this viewpoint was not without its detractors at the time [10].

\section{Current Investigations}

The provision of further evidence for the addictive quality of some foods can be largely credited to the so-called obesity 'epidemic' with its considerable economic burden on a global scale $[11,12]$. At the beginning of the 21 st century, there was a great increase in obesity research worldwide. There was also a noticeable shift from earlier studies that had focused on health-related consequences of obesity, to a new emphasis on the causal factors leading to obesity. Several important technologic advances gave rise to this burgeoning field of research including the Human Genome Project, and the development of sophisticated brain-imaging techniques [13••] for a review]. During this time, scientists, healthcare professionals, and political advocates also began to expose the dramatic changes that had taken place in the food environment, and to draw attention to the food and beverage industry's powerful contribution to poor nutritional habits. In little more than a generation, highly processed foods laced with added sugars, fats, and salt had proliferated in the grocery aisles $[14,15]$. In a recent publication, we demonstrated the impact of this new field of 'food science' by showing the steeply exponential rise in scientific publications related to "food addiction" from 2008 to the present, which followed a relatively barren period of such research in the earlier part of the decade $[16 \bullet \bullet$.

The recent compelling, and non-subjective, evidence of the addictive properties of hyper-palatable foods has come largely from well-controlled and replicated animal models and from human brain-imaging studies.

\section{Preclinical Research}

Experimental studies with rats and mice have demonstrated conclusively that when rodents are chronically exposed to sugar solutions, they display neuropharmacologic and behavioral effects similar to those fostered by many drugs of abuse including physical and behavioral signs of withdrawal, cravings (as indicated by increased motivation to acquire sugar after abstinence), cross-sensitization to other drugs, and enhancement of dopamine in the nucleus accumbens. Several comprehensive reviews have summarized this important body of work [17••, 18, 19]. A recent study has extended this research to planarians - the earliest-evolved animal with a central nervous system - and found that sucrose also produced withdrawal responses and reinforcing effects in an 
invertebrate animal, suggesting that the rewarding consequences of sweeteners are highly conserved across species [20]. Furthermore, in a persuasive series of related experiments, rats who were offered a choice between sucrose (or saccharin) and cocaine self-administration, developed a strong and persistent preference for the former [21•]. In addition, these studies provide strong evidence that sugar reward is more robust and sustainable than cocaine reward, as indicated by its resistance to various genetic, pharmacologic, and neurobiologic interventions [21•].

Preclinical research has also investigated the biobehavioral effects of fatty foods. After a 12-week exposure, rats fed a palatable high-fat diet demonstrated more anxiety- and depressive-like behaviors compared with those on an ingredient-matched low-fat diet [22], and their corticosterone levels were significantly elevated after acute restraint stress. This study also identified neural adaptations that may promote depressive behavior via reduced dopamine tone in the mesolimbic brain pathway. Similarly, other rodent studies have demonstrated down-regulation of dopamine receptor densities in rodents after long-term exposure to highly palatable foods [23], as well as significantly reduced dopamine levels in the nucleus accumbens - as assessed by microdialysis procedures - after 5-day exposure to a high-fat diet in obese-prone rats compared with their obese-resistant counterparts [24].

\section{Human Neuroscience Research}

Neuroimaging studies have demonstrated convincingly that in many individuals - especially those prone to binge eating and obesity - highly palatable food elicits regional brain and behavioral responses similar to those produced by drugs of abuse $[25,26]$. For example, in response to food stimulation, those with a binge eating disorder (BED) released more striatal dopamine than their non-bingeing counterparts [27]. Using magnetic resonance imaging (MRI) procedures, a high glycemic-index test meal also produced significantly greater brain activity in the right nucleus accumbens in obese individuals compared with a low glycemic-index meal [28].

Brain imaging research has also provided good evidence that palatable food cues elicit the same hyper-reactive response in the reward circuitry as drug-related cues [29-31], and that great variation exists in the magnitude of these responses across individuals [32]. Much of the neuroimaging work has focused on functioning in prefrontal cortical regions and its modulation of mesolimbic addictive responses to food. A recent meta-analysis of functional MRI studies found that the prefrontal areas linked to cognitive evaluation processes (e.g., of rewarding stimuli) were most consistently activated in response to food images, and these responses were strongest in those with obesity [33]. By contrast, prefrontal regions associated with cognitive control showed reduced activation.

\section{A Feed-Forward Process of Pathology}

In summary, while highly processed foods do not produce the inebriation caused by alcohol, or the euphoria one can experience from certain stimulant drugs such as cocaine, there are nevertheless pronounced similarities with conventional addictive drugs [16••]. For instance, both have the capability of triggering cravings [34, 35]. Some believe that cravings for sweet and fatty foods likely evolved to enhance human energy intake in environments where energy sources were either scare or unpredictable [36]. Both addictive drugs and hyperpalatable foods are also associated with compulsive consumption, even when the consequences are knowingly dire, and with the inability to cut down [37]. While the parallels are indeed impressive, there is also good evidence that neural responses to hyper-palatable food are more widespread than they are to addictive drugs - especially in medial temporal and superior frontal regions functionally connected to the dorsal striatum [38].

Similar to the downwardly spiraling process of drug addiction, "potentially circular relationships" can be described among the intake of high sugar/fat/salty foods, overeating, and obesity [39]. Excessive stimulation of a core mechanism of human survival - the brain's common reward pathway diminishes our ability to function with a well-regulated capacity for reason, and with the ability to make adaptive decisions in life. In turn, the effects of tolerance and reduced inhibitory control from excessive food intake may prompt more frequent and more prodigious consumption, fostering an interdependent series of behaviors that become more severe and more compulsive over time. It is also well established - as with drugs of abuse — that biologically-based individual differences shape our responses to food from a very early age, and interact importantly with environmental factors, indeed even in utero events, to determine our risk for overeating $[13 \bullet \bullet$.

Finding appropriate treatment and restorative measures for individuals who suffer from pathologic overeating is an important translational aspect of this field of research. Developing behavioral strategies that recognize changes in motivation as a consequence of extreme food consumption are particularly important for many reasons, not least of which are because the consequent brain neuro-adaptations increase the likelihood of using and abusing other addictive substances [40]. From a treatment perspective, we have recently learned of the importance of taking account of seasonal variation in the symptoms of binge eating and addictive behaviors to provide appropriate coping strategies for those who have pronounced negative changes in mood and behavior during the dark months of the year [41]. 


\section{Treatment Considerations}

For clinicians working with individuals who present with compulsive overeating symptoms, it may be useful to consider the treatment implications of the neurobiologic and behavioral parallels between addictive disorders and compulsive overeating [42]. There are currently no empirically supported guidelines for the treatment of "food addiction". There is, however, considerable empirical support for the efficacy of cognitive behavioral therapy (CBT) in the treatment of both BED, a disorder characterized by compulsive overeating, and addiction disorders [43-45]. Indeed, the CBT approach for BED, and the CBT approach for substance abuse, share many similar features including identifying triggers for problem behaviors, developing alternative/distracting behaviors for managing urges, moderating dichotomous thinking, and relapse prevention strategies [46-48]. More recently, motivational interviewing, an approach originally used to enhance motivation for change in substance use disorders, has shown some success in improving outcome in BED [49].

The following treatment considerations are meant to address features of food addiction among overweight individuals presenting with symptoms of binge eating, including intense food cravings, difficulties with inhibitory control, and emotionally-driven overeating. They are not, however, intended for patients with bulimia nervosa $(\mathrm{BN})$ or anorexia nervosa (AN). Although binge eating is a core feature of $\mathrm{BN}$ and occurs in about half of the cases of AN, the binge eating typically occurs in the context of strict dieting and normal or low weight among people with $\mathrm{AN}$ and $\mathrm{BN}$. This is in stark contrast with the clinical presentation of people with BED who typically overeat and binge eat in the absence of food deprivation, and are usually overweight or obese [47]. As discussed by Wilson [50], BN and AN require a different treatment approach that has been well described and empirically validated, an approach that requires reduced dietary restraint and/or restriction [51, 52]. Because individuals with BED do not report elevated dietary restraint or restriction between binge episodes, a different approach is required.

As reviewed earlier in this paper, there is growing evidence that certain types of highly processed foods or certain ingredients in these foods appear to "hijack" the brain of vulnerable individuals in a manner similar to addictive drugs [53]. Typically, it is these types of foods that are consumed during binge episodes among people with compulsive overeating problems. Of course, not everyone with BED meets criteria for food addiction and not everyone with food addiction meets criteria for BED. However, given the behavioral and neurobiologic similarities between drug addiction and excess consumption of hyper-palatable, highly processed foods, integrating an addiction perspective into current treatments for compulsive overeating is likely to prove helpful.
Regarding the treatment of obesity, compulsive overeating is only one factor involved in the etiology of obesity. Approximately half of treatment-seeking obese people meet diagnostic criteria for BED and about half of these meet the Yale Food Addiction Scale (YFAS) criteria for food addiction [54•]. Gearhardt and colleagues [55] have convincingly argued that the application of public health policies, which have been successfully used to control the availability and use of addictive drugs, should be applied to control addictive foods. However, successful implementation of a public-health approach to "food addiction" is likely to involve a lengthy process and such efforts do not obviate the need to develop effective individual treatment strategies.

\section{Psychoeducation}

People who suffer from compulsive overeating problems are often highly self-critical and report high levels of shame related to their disordered-eating behaviors [56]. Providing psychoeducation about the neurobiologic and behavioral parallels between addiction disorders and compulsive overeating may be helpful. Understanding that they may be fighting a strong neurobiologic drive to overeat in an environment that exploits these vulnerabilities could foster a therapeutic sense of self-compassion. It may also help them accept that treatment is likely to involve enduring efforts to resist urges to overeat and to prevent relapse.

\section{Managing Food Cravings and Difficulties with Inhibitory Control}

A core feature of food addiction among overweight individuals with BED is intense food cravings in response to various conditioned cues and strong corresponding urges to overeat despite being sufficiently nourished and, in most cases, overweight or obese [57]. Such individuals report food preoccupation in the absence of food deprivation, and exhibit strong physiologic responses to anticipatory food cues, including thoughts or images of food [58•]. This process is akin to the development of withdrawal symptoms in drug addiction. Thus, to interrupt compulsive-overeating symptoms, individuals with food-addiction symptoms need to develop strategies to increase their ability to sit with food cravings and inhibit urges to overeat. A strategy adapted from Marlatt and Gordon's [59] addiction-treatment manual can be helpful in achieving this goal. This strategy involves employing acceptance-oriented imagery to cope with urges, termed "urge-surfing", to teach clients that cravings will subside if not acted upon. A recent study found evidence that a brief guided-imagery intervention designed to interrupt elaboration of involuntary food thoughts was associated with reduced food cravings [60]. Similarly, mindfulness-skills training may be helpful for increasing a detached awareness of food 
cravings and urges to overeat [61]. In addition, distresstolerance skills training - designed to help suffers tolerate food cravings and inhibit urges to overeat impulsively in response to food cravings - is likely to be of central importance in the treatment of food addiction [61]. Another potentially helpful strategy, adopted from substance-abuse treatment, is cue exposure with response prevention [62]. Treatment consists of repeated exposure to conditioned food cues, but eating (the response) is prevented. There is evidence from various small-scale studies that this approach results in reduced cravings and urges among binge eaters [62].

Pharmacotherapy represents another promising avenue for the development of novel treatments for food addiction. There are several medications available for each of the major classes of addictive drugs which, in conjunction with psychosocial interventions, have been shown to be effective in reducing the likelihood of relapse by reducing urges and cravings [63]. An example of an anti-craving medication is naltrexone, which works by blocking opiate receptors that regulate the release of dopamine in the reward pathways [64]. Although some researchers have suggested that naltrexone might be useful in the treatment of BED [65], placebo-controlled randomized trials have yet to be conducted.

\section{Dealing with Loss of Control}

An inability to reduce consumption despite a strong desire to do so and despite negative consequences is a hallmark of both compulsive overeating and addiction. In drug dependence, loss of control is indicated by the frequent consumption of a substance in larger quantities or over longer periods of time than intended [2]. In BED, loss of control is part of the definition of a binge and refers to a sense that one cannot control what or how much one is eating [2]. According to Volkow and Wise [66], the consumption of hyper-palatable, highly processed foods is likely to have a priming effect that can trigger problematic overeating among vulnerable individuals. Encouraging overweight individuals with BED who report symptoms of food addiction to try and avoid hyperpalatable, highly processed foods may help reduce or eliminate the occurrence of this priming effect. Like drug abuse, compulsive overeating is associated with impulsivity and impaired decision making [67-69]. A strategy, often used in the treatment of drug addiction, aimed at reducing impulsive responding and improving decision-making skills is the use of guided imagery to increase awareness of the likely negative consequences of problematic use [70].

\section{Addressing Emotionally Driven Food Consumption}

People with BED and food addiction report higher levels of depression, and emotion dysregulation than people with BED who do not meet criteria for YFAS food addiction [69]. They also report more severe and more frequent episodes of binge eating, food cravings, emotionally driven eating, addictive personality traits, and impulsive behaviors, prompting the conclusion that co-morbid food addiction reflects a more severe and more compulsive form of BED [13••]. The rewarding effects of hyper-palatable food may be used to increase positive feelings or sensations and to modulate negative emotions [71]. For instance, Udo, Grilo, and Brownell [72] have shown that negative mood states in combination with food cravings reduced the ability of obese individuals to resist eating high-calorie foods. Therefore, the development of emotion-regulation skills, as discussed by Chen and Safer [61], is likely to play an important role in the effective treatment of compulsive overeating.

\section{Enhancing and Maintaining Motivation to Change}

Another challenge common to the treatment of both addictive disorders and compulsive overeating is enhancing and maintaining motivation to change. Motivational interviewing (MI) is a person-centered counseling style that grew out of the recognition that individuals with addictive disorders are often ambivalent about change and reluctant to give up their addictive behaviors [73]. The goal of MI is to resolve ambivalence, enhance motivation to change, and improve treatment outcome. This approach has been applied to other clinical populations including BED with promising results [49]. Anecdotally, individuals with compulsive-overeating problems commonly report that they find it difficult to remain committed to abstaining from overeating when they are experiencing intense cravings. This is likely to be related to an interaction between the widespread neural changes associated with excessive consumption of hyper-palatable, highly processed foods and the challenges inherent in interrupting deeply ingrained learned habits [38]. Thus, motivational-enhancement strategies, designed to maintain abstinence from overeating during the moment-to-moment challenge of coping with intense cravings, is an important aspect of treatment for foodaddiction symptoms among overweight individuals presenting with BED.

\section{Conclusion}

In summary, the compelling neurobiologic and behavioral parallels between compulsive overeating and conventional drug addiction suggest that integrating an addiction perspective into current treatments for compulsive overeating is likely to prove helpful. In this paper, we reviewed the evidence that processed foods high in sugar, fat, and salt have the potential to cause a state of dependence in certain susceptible individuals, and to foster excessive consumption with the feeling of loss of control. We also discuss possible treatment strategies to 
address features of food addiction among overweight individuals presenting with BED including intense food cravings, difficulties with inhibitory control, loss of control over eating, emotionally driven overeating, and maintaining motivation to change.

\section{Compliance with Ethics Guidelines}

Conflict of Interest Caroline Davis and Jacqueline C. Carter declare they have no conflict of interest.

Human and Animal Right and Informed Consent This article does not contain any studies with human or animal subjects performed by either of the authors.

\section{References}

Papers of particular interest, published recently, have been highlighted as:

- Of importance

-. Of major importance

1. American Psychiatric Association. Diagnostic and statistical manual. 5th ed. Washington: American Psychiatric Association Press; 2013.

2. Weiner B, White W. The Journal of Inebriety (1876-1914: history, topical analysis, and photographic images). Addiction. 2007;102: 15-23.

3. Clouston TS. Diseased cravings and paralyzed control: dipsomania; morphinomania; chloralism; cocainism. J Inebr. 1890;12:203-45.

4. Manktelow EJ. Chocolate, women and empire: a social and cultural history by Emma Robertson. Women's Hist Rev. 2013;22:853-5.

5. Loveman K. The introduction of chocolate into England: retailers, researchers, and consumers, 1640-1730. J Soc Hist. 2013;47:2746.

6. Hetherington MM, Macdiarmid JI. "Chocolate addiction": a preliminary study of its description and its relationship to problem eating. Appetite. 1993;21:233-46.

7. Max B. This and that: chocolate addiction, the dual pharmacogenetics of asparagus eater, and the arithmetic of freedom. Trends Pharmacol Sci. 1989;10:390-2.

8. Bruinsma K, Taren DL. Food or drug? J Am Diet Assoc. 1999;99: 1249-56.

9. Tuomisto T, Hetherington MM, Morris M-F, Tuomisto MT, Turjanmaa V, Lappalainen R. Psychological and physiological characteristics of sweet food "addiction". Int J Eat Disord. 1999;25:169-75.

10. Rogers PJ, Smit HJ. Food craving and food "addiction": a critical review of the evidence from a biopsychosocial perspective. Pharmacol Biochem Behav. 2000;66:3-14.

11. Epstein LH. Commentary: future research directions in pediatric obesity research. J Pediatr Psychol. 1999;24:251-2.

12. Swinburn BA, Sacks G, Hall KD, McPherson K, Finegood DT, Moodie ML, et al. The global obesity pandemic: shaped by global drivers and local environments. Lancet. 2011;378:804-11.

13.•- Davis C. From passive overeating to 'food addiction': a spectrum of compulsion and severity. ISRN Obes. 2013; Article ID 43027, doi.org/10.1155/2013/435027. This paper provides a detailed and comprehensive review supporting the addiction potential of certain foods, the food-addiction construct, and its links with binge eating disorder (BED). Empirical evidence also suggests that when the symptoms of BED are highly compulsive and severe, this condition has strong parallels to drug addiction disorders.

14. Novak NL, Brownell KD. Taxation as prevention and as a treatment for obesity: the case of sugar-sweetened beverages. Curr Pharm Des. 2011;17:1218-22.

15. Pomeranz JL, Brownell KD. Portion sizes and beyond: government's legal authority to regulate food-industry practices. N Engl J Med. 2012;367:1383-5.

16.• Gearhardt A, Davis C, Kushner R, Brownell K. The addiction potential of hyperpalatable foods. Curr Drug Abuse Rev. 2011;4: 140-5. This paper provides compelling evidence that hyperpalatable processed foods - those high in sugar, fat, and salt act on the brain reward pathways in a similar way to conventional addictive drugs, and foster the same neuro-adaptations and behavioral responses as these drugs.

17.• Avena NM, Rada P, Hoebel BG. Evidence of sugar addiction: behavioral and neurochemical effects of intermittent, excessive sugar intake. Neurosci Biobehav Rev. 2008;32:20-39. This paper presents a comprehensive and detailed review of the preclinical evidence that the behavioral and physiological responses to intermittent and excessive intake of sugar are remarkably similar to those elicited by drugs of abuse such as opiates.

18. Avena NM, Bocarsly ME, Hoebel BG. Animal models of sugar and fat bingeing: relationship to food addiction and increased body weight. Methods Mol Biol. 2012;829:351-65.

19. Corwin RI, Avena NM, Boggiano MM. Feeding and reward: perspectives from three rat models of binge eating. Physiol Behav. 2011;104:87-97.

20. Zhang C, Tallarida CS, Raffa RB, Rawls SM. Sucrose produces withdrawal and dopamine-sensitive reinforcing effects in planarians. Physiol Behav. 2013;112-113:8-13.

21. Ahmed SH, Guillem K, Vandaele Y. Sugar addiction: pushing the drug-sugar analogy to the limit. Curr Opin Clin Nutr Metab Care. 2013;6:434-9. This paper presents a review of the compelling evidence that sugar can not only substitute for other addictive drugs such as cocaine, but that sweet solutions can even be more rewarding and attractive to laboratory rats than cocaine.

22. Sharma S, Fulton S. Diet-induced obesity promotes depressive-like behavior that is associated with neural adaptations in brain reward circuitry. Int J Obes. 2013;37:382-9.

23. van den Giessen E, de Bruin K, la Fleur SE, van den Brink W, Booji J. Triple monoamine inhibitor tesofensine decreases food intake, body weight, and striatal dopamine $\mathrm{D} 2 / \mathrm{D} 3$ receptor availability in diet-induced obese rats. Eur Neuropsychopharmacol. 2013;22:290 9.

24. Rada P, Bocarsly ME, Barson JR, et al. Reduced accumbens dopamine in Sprague-Dawley rats prone to overeating a fat-rich diet. Physiol Behav. 2010;101:394-400.

25. van Elburg A, Treasure J. Advances in the neurobiology of eating disorders. Curr Opin Psychiatry. 2013;26:556-61.

26. Michaelides M, Miller ML, Subrize M, Kim R, Robison L, Hurd $\mathrm{YL}$, et al. Limbic activation to novel versus familiar food cues predicts food preference and alcohol intake. Brain Res. 2013;1512:37-44.

27. Wang G-J, Geliebter A, Volkow ND, Telang FW, Logan J, Jayne $\mathrm{MC}$, et al. Enhanced striatal dopamine release during food stimulation in binge eating disorder. Obes (Silver Spring). 2011;19:1601-8.

28. Lennerz BS, Alsop DC, Holsen LM, Stern E, Rojas R, Ebbeling $\mathrm{CB}$, et al. Effects of dietary glycemic index on brain regions related to reward and craving in men. Am J Clin Nutr. 2013;98:641-7.

29. Geliebter A, Ladell T, Logan M, et al. Responsivity to food stimuli in obese and lean binge eaters using functional MRI. Appetite. 2006;46:31-5.

30. Grill HJ, Skibicka KP, Hayes MR. Imaging obesity: fMRI, food reward, and feeding. Cell Metab. 2007;6:423-5. 
31. Stoeckel LE, Weller RE, Cook 3rd E, et al. Widespread rewardsystem activation in obese women in response to pictures of highcalorie foods. Neuroimage. 2008;41:636-47.

32. Garcia-Garcia I, Narberhaus A, Marques-Iturria I, et al. Neural responses to visual food cues: insights from functional magnetic resonance imaging. Eur Eat Disord Rev. 2013;21:89-98.

33. Brooks SJ, Cedernaes J, Schioth HB. Increased prefrontal and parahippocampal activation with reduced dorsolateral prefrontal and insular cortex activation to food images in obesity: a metaanalysis of fMRI studies. PLoS ONE. 2013;8:e60393.

34. Pelchat ML, Johnson A, Chan R, et al. Images of desire: food craving activation during fMRI. Neuroimage. 2004;23:1486-93.

35. White MA, Whisehunt BL, Williamson DA, et al. Development and validation of the food-craving inventory. Obes Res. 2002;10:107-14.

36. Gerber LM, Williams GC, Gray SJ. The nutrient-toxin dosage continuum in human evolution and modern health. Q Rev Biol. 1999;74:273-89.

37. Gearhardt AN, Corbin WR, Brownell KD. Food addiction: an examination of the diagnostic criteria for dependence. J Addict Med. 2009;3:1-7.

38. Tomasi D, Volkow ND. Striatocortical pathway dysfunction in addiction and obesity: differences and similarities. Crit Rev Biochem Mol Biol. 2013;48:1-19.

39. Berthoud H-R, Lenard NR, Shin AC. Food reward, hyperphagia, and obesity. Am J Physiol Regul Integr Comp Physiol. 2011;300: R1266-77.

40. Avena NM, Murray S, Gold MS. Comparing the effects of food restriction and overeating on brain reward systems. Exp Gerontol. 2013;48:1062-7.

41. Davis C. A narrative review of binge eating and addictive behaviors: shared associations with seasonality and personality factors. Front Psychiatry. 2013;4:183

42. Davis C, Carter JC. Compulsive overeating as an addiction disorder: a review of theory and evidence. Appetite. 2009;53:1-8.

43. Carroll KM, Onken LS. Behavioral therapies for drug abuse. Am J Psychiatry. 2005;162:1452-60.

44. Wilson GT, Wilfley DE, Agras WS, Bryson SW. Psychological treatments for binge eating disorder. Arch Gen Psychiatry. 2010;67:94-101.

45. Wilson GT, Fairburn CG. Treatment for eating disorders. In: Nathan PE, Gorman JM, editors. A guide to treatments that work. 3rd ed. New York: Oxford University Press; 2007. p. 579-609.

46. Fairburn CG, Marcus MD, Wilson GT. Cognitive-behavioral therapy for binge eating and bulimia nervosa: a comprehensive treatment manual. In: Fairburn CG, Wilson GT, editors. Binge eating. New York: Guilford Press; 1993. p. 361-404.

47. Marcus MD. Adapting treatment for patients with binge-eating disorder. In: Garner DM, Garfinkel PE, editors. Handbook of treatment for eating disorders. 2nd ed. New York: Guilford Press; 1997. p. 484-93.

48. McCrady BS. Alcohol use disorders. In: Clinical handbook of psychological disorders: a step-by-step treatment manual. 4th ed. New York: Guilford Press; 2008. p. 492-546.

49. Vella-Zarb RA, Mills JS, Westra HA, Carter JC, Keating L. A randomized controlled trial of motivational interviewing for binge eating. Int J Eating Disord, in press.

50. Wilson GT. Eating disorders, obesity and addiction. Eur Eating Disord Rev. 2010;18:341-51.

51. Wilson GT, Fairburn CG, Agras WS. Cognitive-behavioral therapy for bulimia nervosa. In: Garner DM, Garfinkel PM, editors. Handbook of treatment for eating disorders. 2nd ed. New York: Guilford Press; 1997. p. 67-93.

52. Pike KM, Carter JC, Olmsted MP. Cognitive behavior therapy for anorexia nervosa. In: Grilo CM, Mitchell JE, editors. The treatment of eating disorders: a clinical handbook. New York: Guilford Press; 2010. p. 83-107.

53. Gearhardt AN, Brownell KD. Can food and addiction change the game? Biol Psychiatry. 2013;73(9):802-3.
54. Gearhart AN, White MA, Masheb RM, Morgan PT, Crosby RD, Grilo CM. An examination of the food addiction construct in patients with binge eating disorder. Int J Eat Disord. 2012;45(5):657-63. This study found that patients with binge eating disorder (BED), who also met criteria for food addiction, reported significantly higher levels of psychiatric disturbance and more frequent binge-eating behavior than BED patients who did not meet criteria for food addiction.

55. Gearhardt AN, Bragg MA, Pearl RL, Schvey NA, Roberto CA, Brownell KD. Obesity and public policy. Annu Rev Clin Psychol. 2012;8:405-30.

56. Goss K, Allan S. Shame, pride and eating disorders. Clin Psychol Psychother. 2009;16:303-16.

57. Davis C, Levitan RD, Reid C, Carter JC, Kaplan AS, Kennedy JL. Dopamine for 'wanting' and opioids for 'liking': obese adults with and without binge eating. Obesity. 2009;17:1220-5.

58. Gearhardt AN, Yokum S, Orr PT, Stice E, Corbin WR, Brownell KD. Neural correlates of food addiction. Arch Gen Psychiatry. 2011;68:808-16. This paper presents data from a functional magnetic resonance imaging study showing that individuals with elevated food-addiction scores exhibited increased neural activation in brain reward circuitry in response to food cues, and reduced activation in inhibitory regions in response to food intake.

59. Marlatt G, Gordon J. Relapse prevention: maintenance strategies in the treatment of addictive disorders. New York: Guilford; 1985.

60. Hamilton J, Fawson S, May J, Andrade J, Kavanagh DJ. Brief guided imagery and body scanning interventions reduce food cravings. Appetite. 2013;71:158-62.

61. Chen EY, Safer DL. Dialectical behavior therapy for bulimia nervosa and binge-eating disorder. In: Grilo CM, Mitchell JE, editors. The treatment of eating disorders: a clinical handbook. New York: Guilford Press; 2010. p. 294-316.

62. Jansen A. A learning model of binge eating: cue reactivity and cue exposure. Appetite. 1998;36:257-72.

63. O'Brien CP. Anticraving medications for relapse prevention: a possible new class of psychoactive medications. Am J Psychiatry. 2005;162:1423-31.

64. Gonzales RA, Weiss F. Suppression of ethanol-reinforced behavior by naltrexone is associated with attenuation of ethanol-induced increase in dialysate dopamine levels in the nucleus accumbens. $\mathbf{J}$ Neurosci. 1998;18:10663-71.

65. De Zwaan M, Mitchell JE. Opiate antagonists and eating behavior in humans: a review. J Clin Pharmacol. 1992;32:1060-72.

66. Volkow ND, Wise RA. How can drug addiction help us understand obesity? Nat Neurosci. 2005;8:555-60.

67. Davis C, Levitan RD, Carter JC, Kaplan AS, Reid C, Curtis C, et al. Personality and binge eating disorder: a case-control study. Int $\mathrm{J}$ Eating Disord. 2008;41:243-50.

68. Davis C, Levitan RD, Muglia P, Bewell C, Kennedy JL. Decisionmaking deficits and overeating: a risk model for obesity. Obes Res. 2004;12(6):929-35.

69. Gearhart AN, White MA, Masheb RM, Morgan PT, Crosby RD, Grilo CM. An examination of the food addiction construct in patients with binge eating disorder. Int J Eat Disord. 2012;45(5):657-63.

70. McGrady BS. Alcohol use disorders: In: Clinical handbook of psychological disorders: a step-by-step treatment manual. 4th ed. New York: Guilford Press, 2008. pp. 492-546.

71. Berg KC, Peterson CD, Crosby RD, Cao L, Crow SJ, Engel SG, et al. Relationship between daily affect and overeating-only, loss of control eating-only, and binge eating episodes in obese adults. Psychiatry Res. 2014;215(1):185-91.

72. Udo T, Grilo CM, Brownell KD, Weinberger AS, Dileone RJ, McKee SA. Modeling the effects of positive and negative mood on the ability to resist eating in obese and non-obese individuals. Eat Behav. 2013;14(1):40-6.

73. Miller WR, Rollnick S. Motivational interviewing: preparing people for change. 2nd ed. New York: Guilford Press; 2002. 